\title{
Fator de Desconto Estocástico no Mercado Acionário Brasileiro
}

\author{
- ANDRÉ Borges CATALÃo*
}

- JOE AKIRA YOSHINO**

\begin{abstract}
RESUMO
Este trabalho implementa as fronteiras de variância mínima para o fator de desconto estocástico, conforme Hansen e Jagannathan (1991) e Cochrane e Hansen (1992), no mercado acionário brasileiro. São consideradas duas abordagens em termos dos retornos das ações e dos prêmios das ações: o Equity Premium Puzzle e o Low Interest Rate Puzzle em face destas metodologias. Adicionalmente, aplicamos o teste econométrico de Burnside (1994) nestes casos. Verificamos que a primeira abordagem produz um fator de desconto estocástico inválido no caso brasileiro. Por outro lado, a formulação com base no prêmio, conforme Cochrane e Hansen (1992), não invalida o fator de desconto estocástico. Assim, não identificamos estes dois puzzles no mercado acionário brasileiro. De fato, o chamado "equity premium puzzle" tem que satisfazer estes dois critérios. Neste sentido, este puzzle não se verifica no mercado acionário brasileiro.
\end{abstract}

\section{Palavras-chave}

prêmio de risco, preço do ativo, mercado de risco, "equity premium risk", "low interest rate puzzle", aversão ao risco, ativos contingentes de Arrow-Debreu, teoria de apreçamento de ativos de Lucas

\section{ABSTRACT}

This article implements the minimum variance frontier for the stochastic discount factor, according to both Hansen and Jagannathan (1991) and Cochrane and Hansen (1992), for the Brazilian stock market. Two approaches are considered in terms of equity returns and equity premium, respectively, the Equity Premium Puzzle and the Low Interest Rate Puzzle. Furthermore, we apply also the econometric test of Burnside (1994) in these approaches. The criteria based on equity return results in an invalid discount factor. On the other hand, the approach using the equity premium, according to Cochrane and Hansen (1992), does not reject the discount factor. Thus, we do not corroborate these two puzzles for the Brazilian equity market. In fact, the equity premium puzzle has to satisfy both criterias above. Thus, in these sense this puzzle does not happen in the Brazilian stock market.

\section{KEY WORDS}

risk premium, asset pricing, market risk, equity premium risk, low interest rate puzzle, risk aversion, Arrow-Debreu contingent claim, Lucas' asset pricing

JEL ClASSIFICATION

D9I, E2, E60, G0, GII, GI2, GI3, H2, H55

* Prof. FEA-IME: Finanças. Banco Santander Banespa. E-mail: abcatalao@santanderbanespa.com.br.

* FEA-USP, Departamento de Economia. Professor Associado em Mercado de Capitais, Renda-Fixa e Derivativos. Webpage: http://www.econ.fea.usp.br/joe. E-mail: pyoshino@usp.br.

Endereço para contato: Rua Indiana, 296, ap. 42. Brooklin, São Paulo, SP. CEP. 04562-000.

(Recebido em maio de 2004. Aceito para publicação em março de 2006). 


\section{INTRODUÇÃO}

Há quase duas décadas Mehra e Prescott (1985) observaram que o valor médio do prêmio de risco das ações norte-americanas, ao longo de quase um século de história, superou o valor que se esperaria levando-se em conta as teorias de ciclo econômico. Para justificar este elevado prêmio de risco, com base no modelo de Lucas (1978), que assume uma função de utilidade do tipo CRRA (constant relative risk aversion), o coeficiente de aversão relativa ao risco $(\alpha)$ teria que ter um valor muito superior ao sugerido por estas teorias. Esse problema foi denominado de Equity Premium Puzzle. Como conseqüência deste valor elevado para $(\alpha)$, Weil (1989) apontou ainda que a taxa de juro livre de risco norte-americana deveria ser bem mais baixa que aquela observada, indicando um Low Interest Rate Puzzle ou Risk-free Rate Puzzle.

O primeiro passo para entender esse fenômeno no contexto da Teoria de Apreçamento de Ativos baseada em consumo intertemporal seria analisar o comportamento da covariância condicional entre o fator de desconto estocástico $(M)$ e a série de retornos das ações. ${ }^{1}$ Isto porque os prêmios exigidos nos investimentos em ativos com risco dependem desta covariância. Uma justificativa aos prêmios elevados seria termos uma covariância condicional bastante negativa, i.e., ativos com baixos pay offs nos estados ruins da natureza. Assim, indivíduos com elevada aversão relativa ao risco $(\alpha)$ exigiriam um enorme prêmio de risco. Desta forma, nos últimos 50 anos observa-se um prêmio de risco de $8 \%$ ao ano nas ações da NYSE com relação do T-Bill.

Uma outra forma de justificar somente parcialmente o enorme prêmio de risco seria propor modificações na função utilidade, seja quanto à forma, seja quanto à introdução de novas variáveis e fricções de mercado, visando introduzir efeitos que aumentem a covariância condicional. Estas abordagens não solucionam o equity premium puzzle. Um ponto crucial é o baixo desvio padrão da taxa de crescimento do consumo com relação à enorme volatilidade dos retornos reais das ações, fazendo com que a covariância entre estas variáveis seja baixa. Neste caso, será necessária uma elevada aversão relativa ao risco para magnificar esta baixa covariância. Mehra (2003) cita vários autores que têm seguido essas linhas de pesquisa. Em termos de formulações alternativas das preferências, podemos citar Abel (1990), Bansal e Yaron (2000), Benartzi e Thaler (1995), Campbell e Cochrane (1999), Constantinides (1990), e Epstein e Zin (1989, 1991). Barberis, Huang e Santos (2001) incluem aversão a perdas na função utilidade e comportamentos passados do investidor (persistência aos hábitos). ${ }^{2}$

1 Veja, por exemplo, Huang e Litzenberg (1988), para uma abordagem mais analítica, ou Cochrane (2001), para um enfoque mais intuitivo.

2 Pesquisas envolvendo mercados incompletos foram desenvolvidas por Constantinides e Duffie (1996), Heaton e Lucas (1997), Mankiw (1986), e Storesletten, Telmer e Yaron (1999). Com relação às imperfeições de mercado, podemos citar Bansal e Coleman (1996), Constantinides, Donaldson e Mehra (2002), Heaton e Lucas (1996), McGrattan e Prescott (2001). 
Hansen e Jagannathan (1991) e, posteriormente, Cochrane e Hansen (1992) mostram que qualquer formulação válida da função utilidade e, por conseqüência, do fator de desconto estocástico $(M)$ do pay off da ação, deve obedecer a uma condição de variância mínima para este fator. Assim, se um fator de desconto estocástico $(M)$ é válido, para um dado valor médio, $E(M)$, podemos atribuir um desvio padrão mínimo $\sigma(M)$ , de modo a gerar uma fronteira de variância mínima no espaço $[E(M), \sigma(M)]$. Esta é a fronteira de Hansen-Jagannathan para o fator de desconto estocástico. Um candidato válido ao fator de desconto estocástico deve gerar um par $[E(M), \sigma(M)]$ na fronteira média-desvio padrão. Cochrane e Hansen (1992) apresentam duas derivações da fronteira de variância mínima para o fator de desconto estocástico com base: i) nos retornos reais das ações; e ii) no prêmio das ações.

Na identificação do US Equity Premium Puzzle \& Low Interest Rate Puzzle, Cochrane e Hansen (1992) utilizam o método da fronteira de variância mínima para o fator de desconto estocástico $(M)$, na formulação de prêmio. Ademais, assumindo uma função de utilidade CRRA (constant relative risk aversion), no caso norte-americano, o coeficiente de aversão relativa ao risco $(\alpha)$ deve ser bastante elevado, da ordem de 40, para gerar pares $[E(M), \sigma(M)]$ referentes a fatores de desconto estocásticos válidos, no interior da fronteira. Este resultado caracteriza o Equity Premium Puzzle. Associado a este valor de $\alpha$, a taxa livre de risco deveria divergir fortemente daquela observada, o que implica o Low Interest Rate Puzzle. ${ }^{3}$ Assim, a importância da variância mínima para o fator de desconto estocástico reside no fato de representar um critério a ser obedecido por qualquer formulação da função utilidade. Além disto, esta técnica pode ser empregada para identificar tanto o Equity Premium, conforme Mehra e Prescott (1985), como o Low Interest Rate Puzzles, segundo Weil (1989). Uma solução suficiente seria satisfazer estes dois puzzles. ${ }^{4}$

Burnside (1994) ressalta que a distância de um par $[E(M), \sigma(M)]$ com relação à fronteira de variância mínima deve ser avaliada econometricamente, apresentando, para tanto, quatro versões de testes relativos a tais distâncias.

No caso brasileiro, vários autores estimam o coeficiente de aversão relativa ao risco $(\alpha)$ e o fator de desconto intertemporal da função utilidade $(\beta)$. Por exemplo, Sampaio (2002) emprega o procedimento de Hansen e Singleton (1983); Bonomo e Domingues (2002) assumem uma função de utilidade proposta por Kreps-Porteus (1978) e usam cadeias de Markov em suas estimações; Issler e Piqueira (2000) esti-

3 A diferença entre a taxa livre de risco observada e aquela requerida pode ser positiva ou negativa, conforme explicado em Campbell et al. (1997, capítulo 8).

4 Contudo, conforme Campbell et al. (1997, capítulo 8) trata-se de uma condição necessária, mas não suficiente. 
mam por GMM a partir de funções utilidade CRRA, Kreps-Porteus e hábito externo, ao passo que Nakane e Soriano (2003) estimam $\alpha$ com moeda na função de utilidade à Sidrauski. Nas análises sobre a fronteira para o caso brasileiro, Soriano (2002) considera somente os retornos das ações e realiza testes segundo a metodologia de Burnside (1994). Adicionalmente, estimações sobre os modelos CAPM e CCAPM podem ser encontradas em Soriano (1999) e Domingues (2000). Outros artigos podem ser mencionados, como Araújo (2002) e Garcia e Olivares (1999).

A contribuição deste artigo no caso brasileiro está em analisar o fator de desconto estocástico sob dois aspectos distintos em termos de retornos das ações e seus prêmios, de modo a aplicar o teste econométrico de Burnside (1994). Analisamos os períodos pré e pós a implementação do Plano Real. ${ }^{5}$ Desta forma, empregamos o procedimento da fronteira de mínima variância do fator de desconto estocástico $(M)$, conforme Hansen e Jagannathan (1991) e Cochrane e Hansen (1992) para o caso brasileiro, utilizando a formulação da fronteira a partir dos retornos das ações e a de prêmios das ações. ${ }^{6}$ A partir de estimações por GMM para o coeficiente de aversão relativa ao risco $(\alpha)$ e o fator de desconto intertemporal da utilidade $(\beta)$ verificamos que a formulação a partir dos retornos de ações produz fatores de desconto estocásticos inválidos, ao passo que a formulação a partir dos prêmios não invalida o fator de desconto estocástico. ${ }^{7}$ A este conjunto de dados aplicamos o teste do tipo III de Burnside (1994) para a distância do par $[E(M), \sigma(M)]$ aos dois tipos de fronteiras. ${ }^{8}$ Com relação ao teste de Burnside, o fator de desconto estocástico $(M)$ é rejeitado como um candidato válido pela fronteira a partir de retornos para a maior parte das estimativas de $\alpha$. O contrário ocorre no caso da fronteira a partir de excesso de prêmios das ações, em que todas as estimativas de $\alpha$ levam a fatores de desconto estocástico válidos. Com relação aos puzzles, estes não foram identificados à luz destes critérios, pois obtemos $2,7 \leq \alpha \leq$ 4,5 por GMM, que é superior ao valor de 0,45 para gerar um par $[E(M), \sigma(M)]$ no interior da fronteira média-variância com base na formulação de prêmio. ${ }^{9}$

O restante do trabalho está dividido da seguinte forma. Na seção 1 são apresentados tanto o modelo de apreçamento de ativos, conforme Lucas (1978), como o método da fronteira média-variância de Hansen e Jagannathan (1991), Cochrane e Hansen

5 Além disso, utilizamos séries de retornos de IBOVESPA com e sem dividendos para compor o retorno das ações a partir dos seus pay offs.

6 Os dados brasileiros são trimestrais e referem-se ao período 1991:1-2003:3.

7 Emprega-se a função de utilidade do tipo CRRA. Os resultados das estimações por GMM encontram-se no apêndice 2 .

8 Burnside (1994) descreve 4 tipos de testes, todos aplicados à fronteira construída a partir de retornos das açôes. Aqui aplicamos o teste do tipo III, que envolve a menor distância do par $[E(M), \sigma(M)]$ à fronteira, sendo o mais restritivo. Além disto, conforme mencionamos, adaptamos o teste para poder ser aplicado à fronteira definida a partir de excessos de retornos.

9 Estimativas do coeficiente de aversão relativa ao risco $(\alpha)$, sob função de utilidade CRRA, para o caso brasileiro, podem ser encontradas em Issler e Piqueira (2000), Nakane e Soriano (2003) e Soriano (2002). 
(1992). Na seção 2 apresentamos os resultados advindos da construção das fronteiras média-variância, nas formulações de prêmio e retornos das ações e discutimos o Equity Premium Puzzle e o Interest Rate Puzzle. A última seção é dedicada às conclusões. Os apêndices I, II, III e IV complementam o trabalho com as fontes de dados, estimações e os procedimentos adotados.

\section{A TEORIA DE APREÇAMENTO DE ATIVOS}

O modelo de apreçamento de ativos baseia-se em Lucas (1978). ${ }^{10}$ Casos particulares desta fórmula são CAPM, APT, Black-Scholes e equity premium puzzle. Neste modelo, um agente representativo resolve um problema de alocação de recursos de modo a obter um fluxo de consumo ótimo dentro de um horizonte de tempo. O processo de decisão é condicionado à informação disponível no presente, representada pela distribuição de probabilidades de ocorrência dos possíveis estados da natureza e depende também da função de utilidade. De forma compacta, o problema de maximização pode ser expresso como:

$$
\operatorname{Max} E_{t}\left[\sum_{j}^{\infty} \beta^{j} U\left(C_{t+j}\right)\right]
$$

em que: $\beta$ é o fator de desconto da utilidade no tempo; $C_{t+j}$ é o consumo real no período $t+j$ e $U\left(C_{t^{+}}\right)$é a utilidade que o investidor obtém em consumir no tempo $t+j$. Adicionalmente, o investidor está sujeito à restrição orçamentária, que lhe permite consumir de acordo com a sua renda e o retorno que é proporcionado pelas ações, nas formas de ganho de capital e recebimento de dividendos. Uma das equações que surgem das condiçóes de primeira ordem é:

$$
P_{k, t} U^{\prime}\left(C_{t}\right)=\beta E_{t}\left[\left(P_{k, t+1}+D_{k, t+1}\right) U^{\prime}\left(C_{t+1}\right)\right.
$$

em que $P_{k, t}$ é preço da ação $k$ no instante $t . D_{k, t}$ é o dividendo pago pela ação $k$ nesse instante. Podemos reescrever esta equação, na forma de retornos:

$$
U^{\prime}\left(C_{t}\right)=\beta E_{t}\left[\left(1+R_{k, t+1}\right) U^{\prime}\left(C_{t+1}\right)\right]
$$

em que o retorno da ação $\left(R_{k, t+1}\right)$, que é dado pelo ganho de capital (variação dos preços das ações) e pelos dividendos recebidos por ação, é dado por:

10 Como referência, podemos citar Huang e Litzenberger (1988, capítulos 5 e 6), Obstfeld e Rogoff (1996, capítulo 5) ou Cochrane (2001). Mehra (1985) apresenta a derivação do modelo em seu artigo. 


$$
1+R_{k, t+1}=\frac{P_{k, t+1}+D_{k, t+1}}{P_{k, t}}
$$

O numerador da equação (4) é o pay off da ação. O lado esquerdo de (3) é o custo marginal de consumir uma unidade monetária real a menos em $t$, ao passo que o lado direito é a satisfação marginal esperada que se obtém ao investir a unidade monetária na ação $k$, no instante $t$, vendendo-a no instante $t+1$, por $1+R_{k, t+1}$ unidades monetárias, e consumindo o resultado. $\mathrm{O}$ investidor iguala o custo marginal da postergação do consumo e o benefício marginal do consumo futuro de modo a obter otimamente o preço da ação. Podemos ainda reescrever (2), definindo o fator de desconto estocástico $M_{t+1} \equiv \beta U^{\prime}\left(C_{t+1}\right) / U^{\prime}\left(C_{t}\right)$, de forma que:

$$
1=E_{t}\left[\left(1+R_{k, t+1}\right) M_{t+1}\right]
$$

Como estamos utilizando uma função de utilidade CRRA, o fator de desconto estocástico coincide com a taxa marginal de substituição intertemporal descontada. Não se pode alterar o coeficiente de aversão relativa ao risco sem alterar a taxa de substituição intertemporal. Além disto, a elasticidade de substituição entre níveis de consumo nos estados contingentes com respeito aos preços de Arrow-Debreu é o inverso do coeficiente de aversão relativa ao risco, $1 / \alpha .^{11,12}$

Tomando a média incondicional de cada lado da equação (5), defasando em um período, para simplificar a notação, podemos escrever, após manipulações simples, com base na definição de covariância:

$$
E\left[1+R_{k, t}\right]=\frac{1}{E\left[M_{t}\right]}\left(1-\operatorname{Cov}\left[R_{k, t}, M_{t}\right]\right)
$$

Em particular, para um ativo livre de risco (riskfree), cuja covariância com o fator de desconto estocástico é zero (ativo com beta zero), temos:

$$
E\left[1+R_{0, t}\right]=1 / E\left[M_{t}\right]
$$

Esta equação possibilita escrever a equação (6) explicitando o prêmio de risco do seguinte modo:

11 Veja, por exemplo, Obstfeld e Rogoff (1996, capítulo 5).

12 Epstein e Zin (1989) separam o coeficiente de aversão relativa ao risco da taxa de substituição intertemporal.

Estud. econ., São Paulo, 36(3): 435-463, jul-set 2006 


$$
E\left[R_{k, t}-R_{0, t}\right]=-E\left[1+R_{0, t}\right] \operatorname{Cov}\left[R_{k, t}, M_{t}\right]
$$

Esta equação sugere que o prêmio de risco (lado esquerdo da equação acima) que é exigido na aplicação num ativo com risco será tanto maior quanto mais negativa for a covariância entre o retorno da ação com o fator de desconto estocástico. ${ }^{13}$

A função de utilidade que será empregada depende somente do consumo, e supõe-se a aditividade intertemporal. Além disto, esta função apresenta coeficiente de aversão relativa ao risco constante (CRRA). ${ }^{14}$

$$
U\left(C_{t}\right)=\frac{C_{t}^{1-\alpha}-1}{1-\alpha}
$$

em que: $0<\alpha<\infty$.

Sob a restrição da função utilidade definida pela equação (9), podemos reescrever a condição (5) como:

$$
1=\beta E_{t}\left[\left(1+R_{k, t+1}\right)\left(\frac{C_{t+1}}{C_{t}}\right)^{-\alpha}\right]
$$

Estimativas da equação (10) usando GMM são apresentadas no Apêndice II.

\subsection{Fronteira Média-Variáncia para o Fator de Desconto Estocástico}

Hansen e Jagannathan (1991) e, posteriormente, Cochrane e Hansen (1992) mostram que há uma fronteira de valores mínimos para o desvio padrão do fator de desconto estocástico $\sigma\left(M_{t}\right)$ em função da sua média $E\left(M_{t}\right) .{ }^{15}$ Há duas formas de representar

13 Suponha que ocorra um estado ruim da natureza amanhã - um baixo consumo e alta utilidade marginal do consumo (correspondendo a um valor alto do fator de desconto estocástico). Para um ativo que deixa de gerar consumo em tal estado, ou seja, um ativo de alto risco, o investidor exige um maior prêmio para postergar o consumo na forma de investimento em ações. Nesta situação, o consumo (baixo) e o retorno exigido (alto) produzem uma covariância muito negativa entre o retorno da ação e o fator de desconto estocástico. Os ativos que pagam em estados bons da natureza, cujos níveis de consumo são altos ou quando a satisfação em consumir uma unidade adicional de consumo é baixa, são menos desejáveis do que aqueles que pagam uma quantidade equivalente quando os estados são ruins. Neste caso, o consumo adicional é mais desejado. Por isso, aqueles ativos são avaliados a um preço menor - o que equivale a dizer que os investidores exigem maior prêmio de risco para adquiri-los. É importante frisar que a satisfação está relacionada ao consumo, e a mesma quantidade de consumo pode gerar diversos níveis de satisfação em diferentes estados da natureza.

14 Esta função torna-se logarítmica quando $\alpha$ vale 1 , que é o limite da equação.

15 Conhecida como fronteira de Hansen-Jagannathan, ou fronteira de média-variância do fator de desconto estocástico. 
esta fronteira neste espaço. A primeira poderia ser gerada pelos retornos dos ativos. A segunda seria gerada pelos prêmios dos ativos de risco com relação ao ativo sem risco (doravante, utilizaremos tanto o termo fronteira de excesso de retorno quanto o termo fronteira de prêmio ao nos referirmos a esta fronteira), apresentando a forma de uma reta a partir da origem. Veremos brevemente o porquê disso. Considere uma regressão do fator de desconto estocástico $\left(M_{t}\right)$ contra uma constante e o vetor $x_{t}$ de retornos reais das ações observados:

$$
M=a+x^{\prime} b+\varepsilon
$$

em que $(a)$ é uma constante e $(b)$ é um vetor de coeficientes angulares e $\varepsilon$ é um ruído branco. Então,

$$
\begin{aligned}
& b=[\operatorname{cov}(x, x)]^{-1} \operatorname{cov}(x, M) \\
& a=E[M]-E\left[x^{\prime} b\right]
\end{aligned}
$$

Supondo que $M$ é um fator de desconto válido, definindo $x \equiv\left(1+R_{k}\right)$, e utilizando a identidade $\operatorname{cov}(x, M) \equiv E(M x)-E(M) E(x)$, a equação (5) fica sendo

$$
\operatorname{cov}(x, M)=1-E(M) E(x)
$$

Substituindo esta equação em (12a), chega-se a:

$$
b=[\operatorname{cov}(x, x)]^{-1}[1-E(M) E(x)]
$$

Portanto, dado $E(M)$, podemos construir $b$, uma vez que temos a série dos retornos reais das ações $(x)$. Como as variáveis independentes da equação (11) são ortogonais aos resíduos, temos:

$$
\operatorname{var}(M)=\operatorname{var}\left(x^{\prime} b\right)+\operatorname{var}(\varepsilon)
$$

Então, $\operatorname{var}\left(x^{\prime} b\right)^{1 / 2}$ é um limite inferior para o desvio padrão de $M$. A fronteira no espaço $E(M)$ versus $\sigma(M)$ tem a forma observada na Figura 1. A região válida para os fatores de desconto estocástico corresponde à região A.

No caso da versão para analisar o prêmio das ações sobre o ativo livre de risco, a regressão (11) é redefinida, agora com a variável $x$ representando o prêmio $\left(R_{k}-R_{0}\right)$. A equação (5) pode ser reescrita como:

$$
0=E\left[M_{t+1}\left(R_{k, t+1}-R_{0, t+1}\right)\right]
$$


Assim, a equação (14) pode ser redefinida como:

$$
b=[\operatorname{cov}(x, x)]^{-1}[-E(M) E(x)]
$$

FIGURA I - FRONTEIRA MÉDIA-VARIÂNCIA: RETORNOS DE AÇÕES

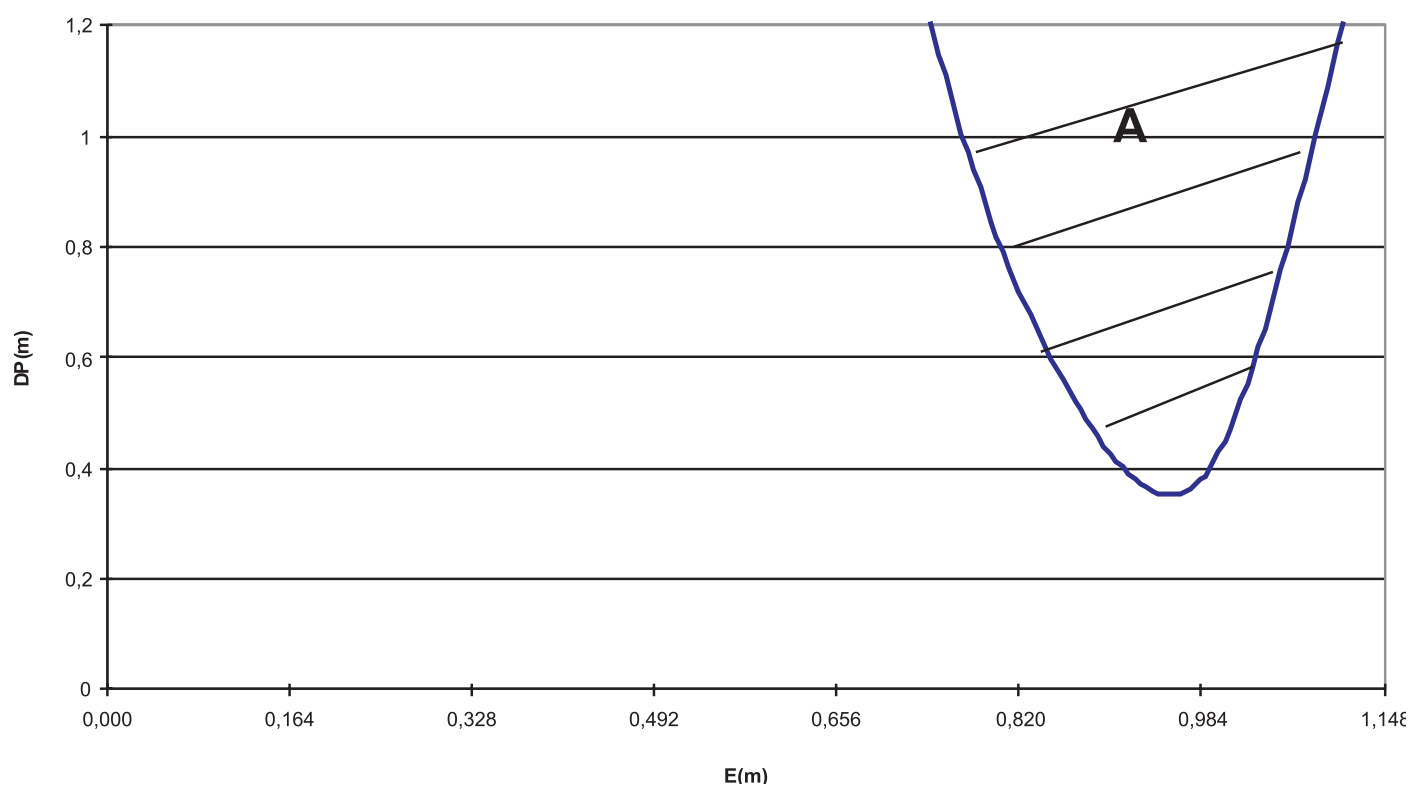

A equação (15) representa, neste caso, uma reta partindo da origem e tocando o mínimo da fronteira definida no caso dos retornos de ações. ${ }^{16}$ A Figura 2 mostra este fato. A região de fatores de desconto válidos para o caso de utilizarmos os excessos de retornos é a região $\mathrm{B}$ (acima da reta), que engloba também a região $\mathrm{A} .{ }^{17}$

16 Para mostrar que de fato a formulação de excesso de retornos gera uma reta, escrevemos a equação do limite da fronteira (15), juntamente com a equação (17):

$\sigma(M) \geq[E(x) / \sigma(x)] E(M)$, em que o termo em colchetes é uma constante.

17 Hansen e Cochrane (1992) aproximam os momentos da população pelas suas definiçõos amostrais, justificando este procedimento com base na estacionariedade, ao menos assintótica, das séries. Como um dos objetivos é identificar a existência dos fatores de desconto válidos, caberia perguntar se o emprego de definições amostrais introduzem erros que alteram as conclusões sobre a validade. Com o objetivo de responder a esta pergunta, Burnside (1994) descreve uma seqüência de quatro testes de Wald. 
FIGURA 2 - FRONTEIRAS MÉDIA-VARIÂNCIA: RETORNOS E PRÊMIOS

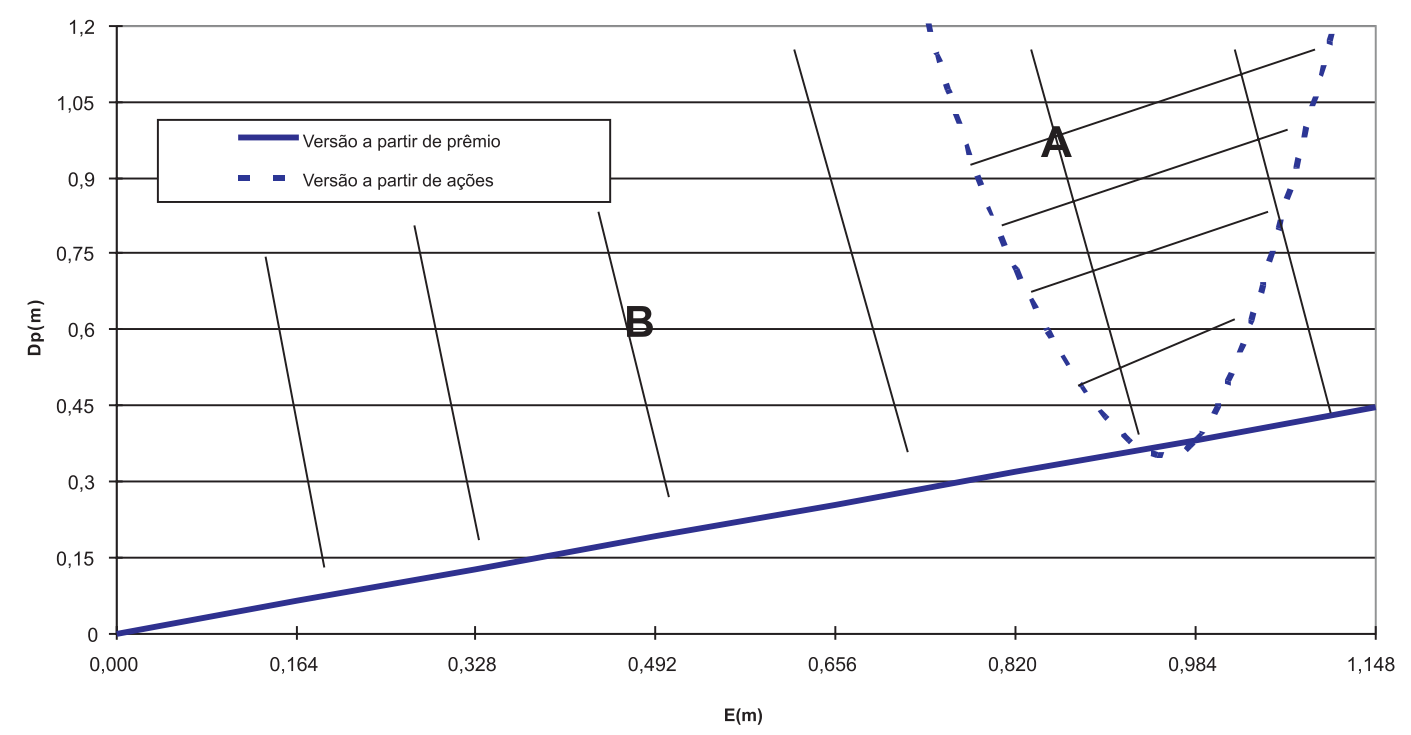

Um modelo de apreçamento que é especificado corretamente deve necessariamente satisfazer aos limites da fronteira média-variância do fator de desconto estocástico. Contudo, esta não é uma condição suficiente, conforme Hansen e Jagannathan (1991).

Cochrane e Hansen (1992) utilizam a fronteira também para identificar o Equity Premium Puzzle, quando reconhecemos que o valor mínimo de $\alpha$ necessário para obter um par $[E(M), \sigma(M)]$ válido é acima daquele obtido em estimações econométricas.

O Interest Rate Puzzle é identificado com base na equação (5). Valores de parâmetros $\alpha$ e $\beta$ que levem a um valor de $E(M)$ incompatível com a média histórica do ativo livre de risco identificaria este Puzzle. Mais especificamente, verificaremos se o valor de $E(M)$, calculado a partir de pares $\alpha$ e $\beta$ necessários para justificar o prêmio observado, implica, pela equação (5), um valor que diverge fortemente da taxa livre de risco média observada em dados históricos.

No Apêndice III descrevemos o procedimento a ser adotado para a construção das fronteiras de média-variância para o fator de desconto estocástico $(M)$, em ambas as formulações. 


\subsection{Testes sobre a Fronteira Média-Variáncia do Fator de Desconto Estocástico}

O Apêndice IV descreve o teste III de Burnside (1994) relacionado à menor distância à fronteira, tanto para a formulação de retorno quanto para a de prêmio. Nesta seção, apresentamos os testes baseados em distâncias à fronteira, segundo Burnside (1994).

A fronteira média-variância é um método visual de validação do fator de desconto estocástico $(M)$ e requer testes sobre as distâncias entre os pontos situados na fronteira e os obtidos por estimaçốes. A distância pode ser medida de forma vertical até a fronteira, ou de forma a se ter a mínima distância. Burnside (1994) propõe quatro tipos de testes de Wald explorando estas possibilidades. ${ }^{18}$ De forma resumida, os testes são:

Teste I: distância vertical à fronteira;

Teste II: distância vertical à fronteira. Contudo, utiliza-se a definição de momento não-centrado. ${ }^{19}$ Este teste é o proposto por Cochrane e Hansen (1992);

Teste III: distância mínima à fronteira, utilizando a definição de momento não-centrado;

Teste IV: distância vertical à fronteira, restringindo o fator de desconto estocástico a valores positivos.

A Figura 3 mostra uma representação geométrica dos testes propostos por Burnside (1994). Neste trabalho utilizaremos o teste do tipo III para avaliar nossas estimações do fator de desconto estocástico ante a fronteira de média-variância. Dos quatro testes propostos, o teste III, segundo Burnside, é o mais restritivo, no sentido de que apresenta maior probabilidade de over-rejection. O risco, então, é rejeitar o candidato a fator de desconto estocástico, quando deveríamos aceitá-lo. O Apêndice IV fornece mais detalhes sobre a construção do teste e mostra como ele pode ser realizado via GMM.

18 Conservadoramente, a hipótese nula é que a distância seja zero. Se rejeitarmos esta hipótese, isto é, se a distância não é nula, o par $[E(M), \sigma(M)]$ pode estar acima ou abaixo da fronteira. Se rejeitarmos o candidato a fator de desconto, estamos admitindo que a distância é menor que zero e que o par estimado está abaixo da fronteira. A distância maior que zero está associada a candidatos a fatores válidos.

19 O estimador do momento não-centrado do fator de desconto estocástico $m$ é definido por $\frac{1}{T} \sum_{t=1}^{T} m^{2}$. No teste I, a definição utilizada é $\frac{1}{T} \sum_{t=1}^{T}(m-\bar{m})^{2}$, em que $\bar{m}$ é a média. 
FIGURA 3 - ILUSTRAÇÃO GEOMÉTRICA DOS TESTES DE BURNSIDE (1994)

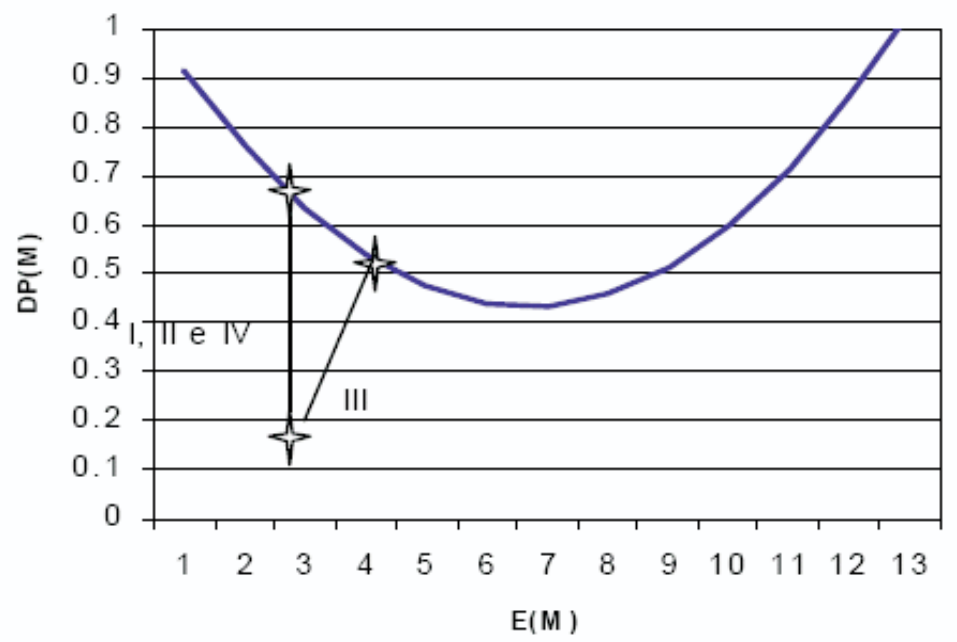

Os quatro tipos de testes mencionados foram construídos em relação à fronteira originada a partir de retornos. Entretanto, Cochrane e Hansen (1992) identificam o Equity Premium Puzzle utilizando a fronteira originada a partir de excessos de retornos (reta a partir da origem). Assim, realizaremos o teste também com respeito a esta última fronteira.

\section{FRONTEIRA MÉDIA-VARIÂNCIA. VALIDAÇÃO DO FATOR DE DESCONTO ESTOCÁSTICO. IDENTIFICAÇÃO DOS PUZZLES}

\subsection{Estimações}

Conforme a teoria apresentada na seção 2, para os dados brasileiros, geramos as fronteiras de média-variância nos casos em que o fator de desconto estocástico é resultado da regressão contra a série de retornos e para o caso da regressão contra o prêmio do retorno das ações sobre o ativo livre de risco (reta). Em ambos os casos, segundo o procedimento apresentado no Apêndice III, as fronteiras são geradas a partir da equação (15), mas no primeiro caso a definição de $b$ é dada pela equação (14), enquanto no segundo caso utiliza-se a equação (17). Ainda segundo a metodologia apresentada no Apêndice III, geramos pares $[E(M), \sigma(M)]$ a partir de um $\beta$ fixo e vários valores 
de $\alpha .{ }^{20}$ Em seguida, segundo o Apêndice IV, aplicamos o teste do tipo III de Burnside (1994) às fronteiras. O Apêndice I descreve os dados utilizados em nossas estimações. Já o Apêndice II apresenta as estimações por GMM.

\subsection{Resultados}

No caso brasileiro, a aplicação do procedimento descrito no Apêndice III foi feita assumindo $\beta=0,95$ e valores de $\alpha$ no intervalo $0,17 \leq \alpha \leq 0,69$. As fronteiras e os pares $[E(M), \sigma(M)]$ gerados a partir deste intervalo de $\alpha$ são apresentados na Figura 4. Esta figura apresenta as fronteiras para ambos os casos, permitindo uma análise visual. Na fronteira construída a partir de retornos reais do índice de ações, nenhum par $[E(M), \sigma(M)]$ gerado a partir dos valores de $\alpha$ considerados está acima da fronteira, o que levaria à rejeição do modelo com função de utilidade tipo CRRA. Entretanto, a fronteira construída a partir de excessos de retornos indica que um valor para o coeficiente de aversão relativa ao risco $(\alpha)$ a partir de 0,45 gera fatores de desconto válidos. A alta rejeição inerente à fronteira gerada a partir de retornos provavelmente se deve à rápida variação do desvio padrão do fator de desconto estocástico $\sigma(M)$ quando sua média $E(M)$ varia, o que gera um formato muito estreito para a fronteira.

Segundo o critério empregado por Cochrane e Hansen (1992), que utiliza a fronteira média-variância na formulação de prêmios, não identificamos o Equity Premium Puzzle, pois o valor mínimo de $\alpha=0,45$ para gerar um par $[E(M), \sigma(M)]$ válido é menor que os obtidos em nossas estimações por GMM. ${ }^{21}$

Com respeito ao Interest Rate Puzzle, a média do fator de desconto estocástico $(M)$ a partir de um valor de $\alpha=0,45$ produz, conforme a equação (7), uma taxa livre de risco de $-5,16 \%$ (ao trimestre). Como esta taxa está abaixo da observada, não temos o Interest Rate Puzzle. ${ }^{22}$ Intuitivamente, considerando o procedimento de Hansen e Singleton (1983), a equação:

$$
R_{f}=-\ln \beta+\alpha E_{t}\left(\Delta c_{t+1}\right)-1 / 2 \alpha^{2} \sigma_{c}^{2}
$$

20 Fixamos $\beta=0,95$.

21 De acordo com o apêndice II, as estimaçóes do coeficiente de aversão a risco $(\hat{\alpha})$ resultou em mediana de 3,62. Issler e Piqueira (2000, tabela l(c), p. 220) estimam uma mediana de 1,70, a partir de dados trimestrais de 1975 a 1994, com variáveis dummy para ajuste de sazonalidade. Nakane e Soriano (2003, tabela 2, p. 17) obtêm o valor de 4,32 para o coeficiente $\hat{\alpha}$, estimando para o período 1991:1 a 2002:1. Vale ressaltar que o trabalho de Nakane e Soriano (2003) volta-se para a análise de moeda. Contudo, um dos parâmetros estimados é o coeficiente de aversão relativa ao risco. Soriano $(2002$, tabela 6A, p. 143) obtém valores de 4,064 a 4,844, com mediana de 4,48 a partir da série de retornos da BOVESPÁ. Bonomo e Domingues (2002, tabela 4, p.112) obtêm o valor de 3,23 para $\hat{\alpha}$.

22 Vale lembrar novamente que esta aplicação da equação (7) utiliza média incondicional. Utilizando o procedimento de Hansen e Singleton (1983) a dados trimestrais de 1980 a 1998, Sampaio (2002) chega a um valor de $-7,4 \%$. 
que apresenta a esperança da variação do log-consumo $\Delta c_{t+1}$, e sua volatilidade, $\sigma_{c}$, indica que com $(\alpha)$ pequeno, alta volatilidade de consumo $(c)$ e baixa perspectiva de consumo (que não compense) o alfa pequeno, a taxa livre de risco, $R_{f}$, fica negativa. No caso do Brasil, quando a fronteira não requer um $\alpha$ elevado, mas, por um outro lado, há alta volatilidade de consumo e baixa perspectiva de consumo, a taxa de juros acaba sendo baixa (aqui, devido aos valores impostos pela fronteira, chegando a ser negativa). Quanto maior a volatilidade do consumo, maior a atitude precaucionária do investidor, reduzindo a taxa de juros necessária. Esta é uma visão da inexistência do puzzle. Vale lembrar que, na verdade, o $\alpha$ estimado é mais alto que o imposto pela fronteira. Assim, a média histórica da taxa livre de risco no Brasil é superior ao requerido pela fronteira de Hansen-Jagannathan. Há um excesso da taxa média observada com relação à requerida.

FIGURA 4 - FRONTEIRA HANSEN-JAGANNATHAN. BR ASIL: 1991:1-2003:3

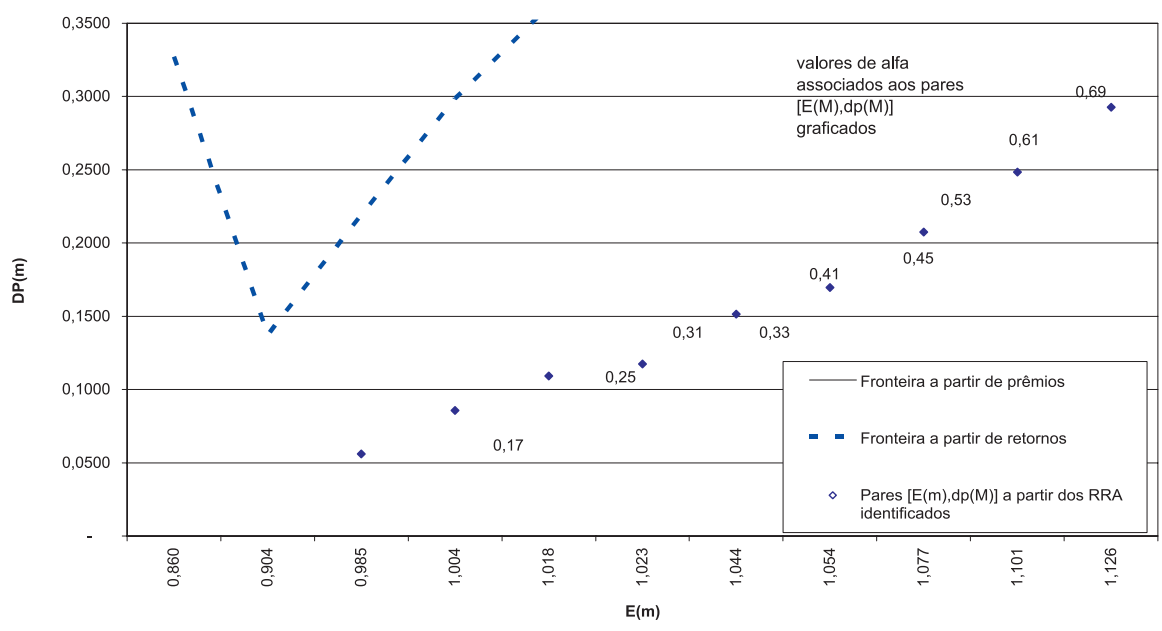

Com relação ao teste III de Burnside (1994) aplicado ao conjunto de estimações do Apêndice II, tem-se que todos os pares a serem testados situam-se entre a fronteira gerada a partir de excessos de retornos e a fronteira gerada a partir de retornos das ações. ${ }^{23,24}$ Assim, quando rejeitamos a hipótese nula de que um par está sobre a fronteira, rejeitamos o candidato válido a fator de desconto estocástico no âmbito da fronteira de retornos, mas o aceitamos no âmbito da fronteira de prêmios. Valores abaixo do nível de confiança de $95 \%$ são considerados sobre a linha da fronteira e valores acima são considerados fora da linha da fronteira.

23 Aplicamos o teste ao conjunto de dados II, que abrange o período 1996:2 a 2003:3, conforme o Apêndice I, porque somente neste período há disponibilidade de dados para o "Indice Bovespa de ações com dividendos".

24 Em outras palavras, os candidatos a fatores de desconto estocástico associados a esses pares são rejeitados pela fronteira gerada a partir de retornos, mas são aceitos pela fronteira gerada a partir de prêmio. 
Os conjuntos de estimações I a IV, apresentados no Apêndice II, para os quais utilizamos o período 1991:1 a 2003:3, tiveram seus valores de coeficiente de aversão ao risco $(\alpha)$ e fator de desconto da função utilidade $(\beta)$ associados a fatores de desconto estocástico $(M)$ de alta volatilidade. Para estes conjuntos, não conseguimos convergência do método GMM na realização dos testes sobre a fronteira. ${ }^{25}$ Assim, apresentamos somente os testes para os conjuntos V a XI, na Tabela 1.

TABELA 1 - NÍVEIS DE CONFIANÇA CALCULADOS A PARTIR DO TESTE SOBRE A FRONTEIR A MÉDIA-VARIÂNCIA. BR ASIL: PERÍODOS II (1996:2-2003:3)

\begin{tabular}{|c|c|c|c|c|c|c|}
\hline Estimação & $\begin{array}{l}\text { Instrumentos (Fronteira } \\
\text { Retorno) }\end{array}$ & $\begin{array}{c}\text { Instrumentos } \\
\text { (Fronteira Prêmio) }\end{array}$ & $\begin{array}{l}\text { Nível de } \\
\text { confiança } \\
\text { (Fronteira } \\
\text { Retorno) }\end{array}$ & $\begin{array}{l}\text { Nível de } \\
\text { confiança } \\
\text { (Fronteira } \\
\text { Prêmio) }\end{array}$ & $\begin{array}{l}\text { Método } \\
\text { (Fronteira } \\
\text { Retorno) }\end{array}$ & $\begin{array}{l}\text { Método } \\
\text { (Fronteira } \\
\text { Prêmio) }\end{array}$ \\
\hline V & $\mathrm{K}, \mathrm{C}(-1), \mathrm{C}(-2), \mathrm{R}$ & $\begin{array}{l}\mathrm{K}, \mathrm{C}(-1), \mathrm{R}(+1) \\
\mathrm{B}(-1)\end{array}$ & 0,990166 & 0,975753 & $\begin{array}{l}\text { ADW } \\
\text { Kernel Bartlett } \\
\text { pw }\end{array}$ & $\begin{array}{l}\text { ADW } \\
\text { Kernel Bartlett } \\
\text { pw }\end{array}$ \\
\hline VI & $K, C(+1), C, R, R(+1)$ & $\begin{array}{l}K, C(-1), R(+1) \\
B(-1)\end{array}$ & 0,954989 & 0,975861 & $\begin{array}{l}\text { NWV } \\
\text { Kernel Bartlett } \\
\text { pw }\end{array}$ & $\begin{array}{l}\text { ADW } \\
\text { Kernel Bartlett } \\
\text { pw }\end{array}$ \\
\hline VII & $K, C, C(-1), R(+1), R, R(-1)$ & $\begin{array}{l}K, C(-1), R(+1) \\
B(-1)\end{array}$ & 0,999099 & 0,975089 & $\begin{array}{l}\text { ADW } \\
\text { Kernel Bartlett } \\
\text { pw }\end{array}$ & $\begin{array}{l}\text { ADW } \\
\text { Kernel Bartlett } \\
\text { pw }\end{array}$ \\
\hline VIII & $\begin{array}{l}K, C(+1), C, C(-1), C(-2) \\
R(+1), R, R(-1), R(-2)\end{array}$ & $\begin{array}{l}K, C(-1), R(+1) \\
B(-1)\end{array}$ & 0,981954 & 0,975886 & $\begin{array}{l}\text { NWV } \\
\text { Kernel Bartlett }\end{array}$ & $\begin{array}{l}\text { ADW } \\
\text { Kernel Bartlett } \\
\text { pw }\end{array}$ \\
\hline IX & $\begin{array}{l}K, C(+1), C, C(-1), R(+1) \\
R, R(-1)\end{array}$ & $\begin{array}{l}\mathrm{K}, \mathrm{C}(-1), \mathrm{R}(+1) \\
\mathrm{B}(-1)\end{array}$ & 0,927149 & 0,975767 & $\begin{array}{l}\text { NWV } \\
\text { Kernel Bartlett }\end{array}$ & $\begin{array}{l}\text { ADW } \\
\text { Kernel Bartlett } \\
\text { pw }\end{array}$ \\
\hline$x$ & $\begin{array}{l}K, C, C(-1), C(-2), C(-3) \\
R(+1), R(-1)\end{array}$ & $\begin{array}{l}K, C(-1), R(+1) \\
B(-1)\end{array}$ & 0,927921 & 0,975830 & $\begin{array}{l}\text { NWV } \\
\text { Kernel Bartlett } \\
\text { pw }\end{array}$ & $\begin{array}{l}\text { ADW } \\
\text { Kernel Bartlett } \\
\text { pw }\end{array}$ \\
\hline XI & $\begin{array}{l}K, C, C(-1), C(-3), R(+1) \\
R(-1)\end{array}$ & $\begin{array}{l}K, C(-1), R(+1) \\
B(-1)\end{array}$ & 0,990415 & 0,975750 & $\begin{array}{l}\text { NWV } \\
\text { Kernel Bartlett } \\
\text { pw }\end{array}$ & $\begin{array}{l}\text { ADW } \\
\text { Kernel Bartlett } \\
\text { pw }\end{array}$ \\
\hline
\end{tabular}

Os resultados mostram que dois conjuntos de dados, IX e X, estão estatisticamente sobre a fronteira de média-variância, na formulação de retornos. Os demais pontos estimados, segundo esta formulação, são rejeitados como candidatos a fatores de des-

25 Embora o método GMM que os gerou tenha sido validado pela estatística $T \times J$, conforme o Apêndice II. 
conto válidos, por estarem na parte inferior da mesma. Todos os conjuntos de dados representam pares $[E(M), \sigma(M)]$ válidos na formulação de excesso de retornos.

Apresentamos estimações para o período antes da implementação do Plano Real (1991:1 a 1994:2) e pós-Plano Real (1994:2 a 2003:3), segundo a Tabela A2 do Apêndice II. Para estes períodos de estimação, a Tabela 2 mostra que os pares $[(E(M), \sigma(M)]$ não se situam sobre a fronteira de Hansen-Jagannathan, tanto na versão de retornos quanto na versão de prêmios. Dado que os pares situam-se entre as fronteiras, concluímos que a fronteira na versão de retornos invalida os candidatos a fator de desconto, ao passo que estes são validados pela fronteira de prêmio.

TABELA 2 - NÍVEIS DE CONFIANÇA CALCULADOS A PARTIR DO TESTE SOBRE A FRONTEIRA MÉDIA-VARIÂNCIA. BRASIL: PRÉ-PLANO REAL (1991:1 A 1994:2) E PÓS-PLANO REAL (1994:2 A 2003:3)

\begin{tabular}{|c|c|c|c|c|c|c|c|}
\hline Estimação & Período Utilizado & $\begin{array}{c}\text { Instrumentos } \\
\text { (Fronteira } \\
\text { Retorno) }\end{array}$ & $\begin{array}{c}\text { Instrumentos } \\
\text { (Fronteira Prêmio) }\end{array}$ & $\begin{array}{l}\text { Nível de } \\
\text { confiança } \\
\text { (Fronteira } \\
\text { Retorno) }\end{array}$ & $\begin{array}{l}\text { Nível de } \\
\text { confiança } \\
\text { (Fronteira } \\
\text { Prêmio) }\end{array}$ & $\begin{array}{l}\text { Método } \\
\text { (Fronteira } \\
\text { Retorno) }\end{array}$ & $\begin{array}{l}\text { Método } \\
\text { (Fronteira } \\
\text { Prêmio) }\end{array}$ \\
\hline Pré-Real & 1991:1 à 1994:2 & $\begin{array}{l}K, C, C(-1), \\
C(-2), C(-5), R, \\
R(-1)\end{array}$ & $\begin{array}{l}\mathrm{K}, \mathrm{C}(-1), \mathrm{R}(+1), \\
\mathrm{B}(-1)\end{array}$ & 0,959537 & 0,978267 & $\begin{array}{l}\text { NWV } \\
\text { Kernel } \\
\text { Bartlett } \\
\text { pw }\end{array}$ & $\begin{array}{l}\text { ADW } \\
\text { Kernel } \\
\text { Bartlett } \\
\text { pw }\end{array}$ \\
\hline Pós-Real & $1994: 3$ à 2003:3 & $\begin{array}{l}K, C, C(-1), \\
C(-2), C(-3), R, \\
R(-1)\end{array}$ & $\begin{array}{l}K, C(-1), R(+1), \\
B(-1)\end{array}$ & 0,999995 & 0,978289 & $\begin{array}{l}\text { ADW } \\
\text { Kernel } \\
\text { Bartlett } \\
\text { pw }\end{array}$ & $\begin{array}{l}\text { ADW } \\
\text { Kernel } \\
\text { Bartlett } \\
\text { pw }\end{array}$ \\
\hline
\end{tabular}

Resumindo, no mercado brasileiro não identificamos a existência dos dois puzzles (equity premium and interest rate). O modelo CRRA da função utilidade é rejeitado no âmbito da fronteira de média-variância do fator de desconto estocástico, na versão dos retornos, para a maior parte dos valores estimados. ${ }^{26}$ Todavia, o modelo é aceito pela versão de excesso de retornos da fronteira, a partir do valor mínimo de 0,45 para o coeficiente de aversão relativa ao risco $(\alpha)$, que inclui os valores das estimações.

\section{CONCLUSÕES}

Este artigo revisita o Equity Premium Puzzle e o Interest Rate Puzzle segundo a metodologia da fronteira média-variância para o fator de desconto estocástico $(M)$ do payoff

26 Vemos aqui a utilidade do teste de Burnside (1994), pois, a priori, teríamos rejeitado todos os pontos se empregássemos somente a análise visual. 
da ação, conforme Hansen e Jagannathan (1991) e Cochrane e Hansen (1992). Utilizamos esta abordagem tanto para os retornos como para os prêmios das ações, implementando a metodologia para o mercado acionário brasileiro (BOVESPA). Nosso critério para identificar os puzzles está relacionado ao coeficiente de aversão relativa ao risco $(\alpha)$ necessário para gerar um par $[E(M), \sigma(M)]$ no interior da fronteira média-variância de Hansen e Jagannathan (1991). A obtenção de valores excessivamente elevados de $\alpha$ (comparativamente a valores considerados razoáveis pelas teorias de ciclo econômico) identificam o Equity Premium Puzzle. Por outro lado, o Interest Rate Puzzle surge das implicaçôes que um $\alpha$ elevado tem para o nível da taxa de juros. O cálculo de $[E(M), \sigma(M)]$ é feito de forma incondicional. Adicionalmente, testamos econometricamente a distância de pares $[E(M), \sigma(M)]$, gerados a partir de estimações por GMM dos coeficientes $\alpha$ e $\beta$, às fronteiras, aplicando o procedimento do teste III de Burnside (1994) à fronteira de retornos e gerando um teste análogo para a fronteira de prêmios.

A fronteira de Hansen-Jagannathan a partir dos retornos das ações exige valores explosivos de $\alpha$ para gerar um fator de desconto estocástico $(M)$ válido. Ou seja, a geometria da fronteira requer uma alta volatilidade $\sigma(M)$ para cada valor de $E(M)$. Visualmente, nenhum par $[E(M), \sigma(M)]$ gerado a partir dos valores do coeficiente de aversão relativa ao risco $(\alpha)$ e do fator de desconto da utilidade $(\beta)$ situou-se em seu interior. Contudo, a aplicação do teste III indicou que parte do conjunto $(\alpha, \beta)$ estimado por GMM produz pares $[E(M), \sigma(M)]$ que podem ser considerados sobre a fronteira e, portanto, são candidatos válidos para o fator de desconto estocástico. Deve-se ressaltar, no entanto, que a maioria dos pares foi rejeitada com respeito à fronteira dos retornos. Por outro lado, de acordo com a fronteira a partir dos excessos de retornos, o valor mínimo que o coeficiente de aversão relativa ao risco $(\alpha)$ deve assumir foi calculado em 0,45 . Com respeito a esta fronteira, a rejeição apontada pelos testes relativos à distância, quando aplicados aos pares $(\alpha, \beta)$ estimados por GMM, leva à hipótese alternativa de que os pares estejam numa região com fatores de desconto estocásticos válidos. Portando, segundo o critério utilizado para este tipo de fronteira, estimativas de $\alpha$ variando de 2,7 a 4,5 indicam não existir o Equity Premium Puzzle no Brasil. ${ }^{27}$ Verificamos que no Brasil não há também o Interest Rate Puzzle, uma vez que a taxa de juros livre de risco associada à $\alpha=0,45$ deveria ser de $-5,16 \%$ ao trimestre, indicando haver um excesso da taxa de juros observada em relação à requerida. Mais ainda: o fator de desconto é rejeitado na formulação de retornos das ações, ao passo que não ocorre rejeição na formulação de excesso de retornos.

Como próximos passos, sugerimos a aplicação da teoria de Hansen e Jagannathan e dos testes de Burnside (1994) a novas formulações da função utilidade que incor-

27 No apêndice II citamos referências que contêm estimações de $\alpha$ para o caso brasileiro. 
porem explicações monetárias. Uma outra linha de pesquisa seria tentar solucionar o puzzle ao identificar variáveis que afetam a covariância entre o fator de desconto estocástico e a taxa de retorno do ativo. Yoshino (2005) sugere a não-neutralidade da moeda (inflação) e bancos de modo a derivar uma outra fórmula de apreçamento de ativos nesta economia.

\section{APÊNDICE I - DADOS UTILIZADOS}

Com relação ao caso brasileiro, utilizamos séries trimestrais para os dados envolvendo o período 1991:1 a 2003:3. ${ }^{28}$ Consideramos dois períodos: o primeiro (I), de 1991:1 a 2003:3; e o segundo (II), de 1996:2 a 2003:3. Para o período I, empregamos a série IBOVESPA ex-dividendo, calculando retornos sobre o índice na forma: ${ }^{29}$

$$
1+R_{k, t+1}=\frac{P_{k, t+1}}{P_{k, t}}
$$

Para o período II, calculamos os retornos sobre o índice tanto na forma da equação (4) quanto na forma da equação (19). Assim, o conjunto de dados para o caso brasileiro é composto das seguintes variáveis:

1) Série trimestral do índice dessazonalizado do Consumo das Famílias, divulgado nas Contas Nacionais, no sistema SIDRA, do IBGE; ;0, 31

2) Série mensal do Índice Geral de Preços - Disponibilidade Interna (IGP-DI), calculado pela FGV, disponível no site do Ipeadata; ${ }^{32}$

3) Índice BOVESPA de ações (ex-dividendo), disponível no provedor Reuters;

28 Com referência ao índice de ações, empregamos o IBOVESPA. A forma como este índice é divulgado não engloba dividendos (ex-dividendo). Contudo, segundo a equação (4), o modo de calcular o retorno que entra na equação de Asset Pricing requer que seja utilizado o pay off no numerador, que corresponderia a usar um índice com dividendos. No entanto, dados sobre dividendos em forma eletrônica só existem a partir do segundo trimestre de 1996.

29 A fórmula de cálculo expressa pela equação (19) é a adotada por Issler e Piqueira (2000).

30 Esta série não distingue consumo de bens duráveis de consumo de bens não-duráveis. $\mathrm{O}$ método de dessazonalização é o X-12 do BEA.

31 Os dados de consumo extraídos do SIDRA e a série de taxas de juros de CDBs são também utilizados por Nakane e Soriano (2003). Issler e Piqueira (2000) utilizam dados das contas nacionais do IBGE, mas fazem ajustes para adequar periodicidades diversas de divulgação aos demais dados. Além disso, utilizam a série do IBOVESPA ex-dividendo, como índice de ações, e o IGP-DI, como deflator. Como série de taxas de juros, Issler e Piqueira (2000) utilizam a série relativa aos títulos federais, publicada pela ANDIMA.

$32 \mathrm{O}$ índice foi acumulado trimestralmente para gerarmos séries reais trimestrais de crescimento de consumo per capita e retornos do índice de ações IBOVESPA.

Estud. econ., São Paulo, 36(3): 435-463, jul-set 2006 
4) Índice BOVESPA de ações com dividendo, disponível no provedor Bloomberg; ${ }^{33}$

5) Série anual de População Total, disponível no sistema SIDRA, do IBGE; 34

6) Série mensal de taxas de juros CDB pré-fixado (\%am), divulgado pela ANDIMA, disponível no site do Ipeadata. ${ }^{35}$

\section{APENNDICE II - ESTIMATIVAS POR GMM}

Neste apêndice apresentamos as estimativas por GMM, para os parâmetros $\alpha$ (coeficiente de aversão ao risco) e $\beta$ (fator de desconto da função utilidade no tempo), a partir da equação (10). ${ }^{36}$ Segundo mencionado no Apêndice I, a análise está dividida em dois períodos: o período I, compreendendo 1991:1 a 2003:3; e o período II, compreendendo 1996:2 a 2003:3, para o qual utilizamos a série do índice IBOVESPA com dividendos. ${ }^{37} \mathrm{~A}$ Tabela Al apresenta os resultados das estimações. Os conjuntos denominados V, VIII e IX utilizaram os retornos das ações calculados segundo a equação (4). Os demais conjuntos empregaram a equação (19), isto é, sem considerar os dividendos.

33 O índice calculado pela Bloomberg (acessado usando a função Ibov <index > TRA) é gerado com base em dados de pagamentos de dividendos pelas empresas que compõem o IBOVESPA. Estes dados estão disponíveis no site da Bovespa, a partir de 1996:2.

34 Utilizamos interpolações lineares para gerar dados trimestrais de população.

35 Acumulamos as taxas, que são divulgadas \%a.m., para obtermos valores trimestrais.

36 Aplicamos o método GMM para diversos instrumentos e períodos, gerando os grupos de estimações I a XI. As variáveis contemporâneas são denotadas por $C(+1)$, que representa $C_{t+1} / C_{t}, \mathrm{e} R(+\mathrm{l})$, que corresponde à $1+R_{k, t+1}$. A notação das defasagens das variáveis instrumentais é coerente com esta notação das variáveis contemporâneas. O grupo II inclui defasagens de retornos reais de dividendos como instrumentos, denotados por $D(+k)=d_{t^{+k}} / d_{t+(k-1)}$. A taxa nominal de juros livre de risco está representada por $B$, nas defasagens $B(+1)$ e B. Todos os grupos de estimação utilizam uma constante $(K)$. As formas funcionais utilizadas para o cálculo do kernel foram a quadrática e a de Bartlett. Os métodos de determinação da largura da banda (bandwidth) empregados foram de Newey-West com largura fixa de banda (NWF), na qual a largura é função do número de pontos. Já os métodos de Andrews (ADW) e Newey-West variável (NWV) empregam autocorrelaçóes dos dados utilizados para a determinação da banda. A técnica de prewithening (pw) ajusta um modelo AR(1) aos resíduos antes de calcular a matriz. Para mais detalhes, veja Newey e West (1987), Andrews (1991) e Newey-West (1994). Sobre estimações por GMM, o leitor pode consultar Hansen e Singleton (1982a) e Hansen e Singleton (1982b).

37 No período I realizamos estimações com o índice IBOVESPA sem dividendos, somente. No período II, por sua vez, realizamos estimações utilizando as séries sem dividendos e com dividendos, para poder comparar resultados. 
TABELA AI - AVERSÃO RELATIVA AO RISCO E FATOR DE DESCONTO INTERTEMPORAL. MÉTODO GMM. BRASIL

\begin{tabular}{|c|c|c|c|c|c|c|}
\hline Estimação & $\begin{array}{l}\text { Período } \\
\text { Utilizado }\end{array}$ & Instrumentos & $\begin{array}{c}\beta \\
\text { (desvio-padrão) } \\
\text { (t-estatístico) }\end{array}$ & $\begin{array}{c}\alpha \\
\text { (desvio-padrão) } \\
\text { (t-estatístico) }\end{array}$ & $\begin{array}{c}\text { p-valor da } \\
\text { estatística T x J }\end{array}$ & Método \\
\hline 1 & 1 & $\begin{array}{l}\mathrm{K}, \mathrm{C}(+1), \mathrm{C}(-1), \mathrm{C}(-6) \\
\mathrm{C}(-8), \mathrm{R}(+1), \mathrm{R}(-3), \mathrm{R}(-5) \\
\mathrm{R}(-6), \mathrm{R}(-8)\end{array}$ & $\begin{array}{c}0,954086 \\
(0,023734) \\
(40,19833)\end{array}$ & $\begin{array}{c}2,701281 \\
(1,042053) \\
(2,592267)\end{array}$ & 0,970229 & $\begin{array}{l}\text { ADW Kernel } \\
\text { Bartlett }\end{array}$ \\
\hline$\|$ & I & $\begin{array}{l}\mathrm{K}, \mathrm{C}(+1), C(-1), C(-6), C(- \\
8), R(-1), R(-6), R(-8)\end{array}$ & $\begin{array}{c}0,95857 \\
(0,020021) \\
(47,87758)\end{array}$ & $\begin{array}{c}4,575938 \\
(1,191845) \\
(3,839372)\end{array}$ & 0,445000 & $\begin{array}{l}\text { VNW Kernel } \\
\text { Bartlett }\end{array}$ \\
\hline III & I & $K, C(-1), C(-5), B, B(+1)$ & $\begin{array}{c}0,972695 \\
(0,027745) \\
(35,05806)\end{array}$ & $\begin{array}{l}2,414232 \\
(0,733384) \\
(3,291908)\end{array}$ & 0,790815 & $\begin{array}{l}\text { ADW Kernel } \\
\text { Bartlett }\end{array}$ \\
\hline IV & I & $K, C(-1), C(-5), B, B(+1)$ & $\begin{array}{l}0,961847 \\
(0,22915) \\
(41,97443)\end{array}$ & $\begin{array}{l}2,892086 \\
(0,977283) \\
(2,959314)\end{array}$ & 0,789502 & $\begin{array}{l}\text { VNW Kernel } \\
\text { Bartlett pw }\end{array}$ \\
\hline V & $\|$ & $\begin{array}{l}\mathrm{K}, \mathrm{C}(+1), \mathrm{C}(-1), \mathrm{C}(-4), \mathrm{R} \\
\mathrm{R}^{\wedge} 2\end{array}$ & $\begin{array}{c}0,943129 \\
(0,020751) \\
(45,45088)\end{array}$ & $\begin{array}{c}3,218362 \\
(1,352134) \\
(2,380209)\end{array}$ & 0,308875 & $\begin{array}{l}\text { VNW Kernel } \\
\text { Bartlett }\end{array}$ \\
\hline $\mathrm{Vl}$ & $\|$ & $\begin{array}{l}K, C(+1), C(-1), C(-4), C(- \\
6), R, R^{\wedge} 2, R(-1)\end{array}$ & $\begin{array}{c}0,958227 \\
(0,018542) \\
(51,67836)\end{array}$ & $\begin{array}{c}4,018672 \\
(1,964202) \\
(2,045957)\end{array}$ & 0,322058 & $\begin{array}{l}\text { ADW Kernel } \\
\text { Bartlett pw }\end{array}$ \\
\hline VII & $\|$ & $\begin{array}{l}K, C(+1), C(-1), C(-4), R \\
R^{\wedge} 2, R(-6)\end{array}$ & $\begin{array}{c}0,947269 \\
(0,017008) \\
(55,6954)\end{array}$ & $\begin{array}{c}3,476748 \\
(1,273326) \\
(2,730477)\end{array}$ & 0,395483 & $\begin{array}{l}\text { VNW Kernel } \\
\text { Bartlett pw }\end{array}$ \\
\hline VIII & $\|$ & $\begin{array}{l}K, C(+1), C(-1), C(-4), R \\
R^{\wedge} 2, B\end{array}$ & $\begin{array}{c}0,955979 \\
(0,027485) \\
(34,78137)\end{array}$ & $\begin{array}{c}4,276759 \\
(2,085264) \\
(2,050944)\end{array}$ & 0,240721 & $\begin{array}{l}\text { ADW Kernel } \\
\text { Bartlett }\end{array}$ \\
\hline IX & II & $\begin{array}{l}K, C(+1), C(-1), C(-4), R \\
R^{\wedge} 2, B\end{array}$ & $\begin{array}{c}0,948069 \\
(0,02028) \\
(46,74926)\end{array}$ & $\begin{array}{c}3,625704 \\
(1,296175) \\
(2,797233)\end{array}$ & 0,333335 & $\begin{array}{l}\text { VNW Kernel } \\
\text { Bartlett }\end{array}$ \\
\hline$x$ & II & $\begin{array}{l}K, C(+1), C(-1), C(-4), C(- \\
6), B(+1), B, R^{\wedge} 2\end{array}$ & $\begin{array}{c}0,96548 \\
(0,027256) \\
(35,42228)\end{array}$ & $\begin{array}{c}4,171685 \\
(1,975233) \\
(2,111996)\end{array}$ & 0,290699 & $\begin{array}{l}\text { ADW Kernel } \\
\text { Bartlett pw }\end{array}$ \\
\hline $\mathrm{XI}$ & $\|$ & $\begin{array}{l}K, C(+1), C(-1), C(-4), C(- \\
6), B(+1), B, R^{\wedge} 2\end{array}$ & $\begin{array}{c}0,927418 \\
(0,024375) \\
(38,04865)\end{array}$ & $\begin{array}{c}3,894788 \\
(1,345619) \\
(2,894422)\end{array}$ & 0,243099 & $\begin{array}{l}\text { VNW Kernel } \\
\text { Bartlett }\end{array}$ \\
\hline
\end{tabular}

Considerando a estatística $T \times J$, a maior evidência contra o modelo ocorre no conjunto de estimação VIII. Com relação ao valor do fator de desconto intertemporal da função utilidade $(\widehat{\beta})$, para os conjuntos relativos ao período I, obtivemos valores entre 0,95 e 0,97 , com mediana 0,96 . Para o período II, os valores ficaram entre 0,93 e 
0,97, com mediana 0,95. Considerando os dois períodos, a mediana foi de 0,96 . As estimações do coeficiente de aversão relativa ao risco $(\hat{\alpha})$, considerando o período I, ficaram entre 2,41 e 4,58, com mediana 2,80. Para o período II, os valores ficaram entre 3,22 e 4,28, com mediana 3,89. Considerando os dois períodos, a mediana foi de 3,626. Vemos, pois, que não houve grande diferença ao empregar a definição de retorno das ações usando a equação (4) ou a equação (19).

Issler e Piqueira (2000, tabela 1(b), p. 219), reportam valores do coeficiente de aversão relativa ao risco $(\hat{\alpha})$ entre 0,891 e 2,202 , significativos a $5 \%$, a partir de dados trimestrais ajustados para sazonalidade, no período 1975-1994. Considerando todos os valores, inclusive os não-siginificativos, a mediana é de 0,62 . No mesmo artigo, a tabela l(c), p. 220 reporta valores entre 1,176 e 6,4381 (mediana de 1,70) para o mesmo período, utilizando variáveis dummy para ajuste de sazonalidade, todos significativos a 1\%. Estimando com dados anuais, a tabela 1(a), p. 218 apresenta valores entre 2,64 e 6,820. Nakane e Soriano (2003, tabela 2, p. 17) obtêm o valor de 4,3209 para o coeficiente $\hat{\alpha}$, estimando para o período 1991:1 a 2002:1. ${ }^{38}$ Soriano (2002, tabela 6A, p. 143) obtém valores de 4,064 a 4,844, com mediana de 4,48 a partir da série de retornos da BOVESPA. ${ }^{39}$ Bonomo e Domingues (2002, tabela 4, p.112) obtêm valor de 3,23 para $\hat{\alpha} .^{40}$

A Tabela A2 mostra estimações por GMM dos coeficientes de aversão ao risco $(\hat{\alpha})$ e do fator de desconto intertemporal da utilidade $(\widehat{\beta})$ para os períodos anterior e posterior ao Plano Real (1991:1 a 1994:2 e 1994:3 a 2003:3, respectivamente). No período pré-Real, obtivemos coeficiente de aversão ao risco $(\hat{\alpha})$ igual a 0,8845 . No período pós-Real, o $\hat{\alpha}$ elevou-se para 2,119. O fator de desconto intertemporal mostrou-se mais baixo no período anterior ao Plano Real relativamente ao período posterior.

38 Apesar do trabalho de Nakane e Soriano (2003) utilizarem o modelo monetário de Sidrauski, um dos parâmetros estimados é o coeficiente de aversão relativa ao risco.

39 Soriano (2002) utiliza dados trimestrais de 1980 a 1998.

40 Os dados utilizados por Bonomo e Domingues (2002) são trimestrais, de 1986 a 1998. Os autores estimam a partir de um modelo de Markov switching e função utilidade Kreps-Porteus (1978). 
TABELA A2 - AVERSÃO RELATIVA AO RISCO E FATOR DE DESCONTO INTERTEMPORAL DA UTILIDADE. MÉTODO GMM. BRASIL: PRÉ E PÓS PLANO REAL

\begin{tabular}{lllcccl}
\hline & $\begin{array}{c}\text { Período } \\
\text { Utilizado }\end{array}$ & \multicolumn{1}{c}{ Instrumentos } & $\beta$ & $\alpha$ & $\begin{array}{c}\text { p-valor da } \\
\text { estatística } \\
\text { Tx J }\end{array}$ & Método \\
& & & $\begin{array}{c}\text { (desvio-padrão) } \\
\text { (t-estatístico) }\end{array}$ & $\begin{array}{c}\text { (desvio-padrão) } \\
\text { (t-estatístico) }\end{array}$ & & \\
\hline Pré-Real & $1991: 1$ à & K, C, C(-2), C(-4), C(- & 0.893730 & 0.884454 & 0,271090 & ADW \\
& $1994: 2$ & 5), R(-2), B, B(-1) & $(0.009462)$ & $(0.153791)$ & & Kernel Bartlett \\
& & & $(94.45010)$ & $(5.751022)$ & & pw \\
Pós-Real & $1994: 3$ à & K, C, C(-2), C(-4), C(- & 0.971105 & 2.119257 & 0,306090 & ADW \\
& $2003: 3$ & $5), \mathrm{R}(-2), B, B(-1)$ & $(0.025213)$ & $(0.429622)$ & & Kernel Bartlett \\
& & & $(38.51663)$ & $(4.932840)$ & & pw \\
\hline
\end{tabular}

\section{APÊNDICE III - METODOLOGIA PARA A IDENTIFICAÇÃO DOS FATORES DE DESCONTO. FRONTEIRA MÉDIA-VARIÂNCIA}

Nesta seção apresentamos o procedimento utilizado para identificar fatores de desconto estocástico válidos, conforme exposto na seção 1.1, com base nas equações (12a) - (17). Inicialmente, ressalta-se que a série usada como regressor do fator de desconto estocástico $(M)$, denotada por $x$, é fixa, representando retornos do índice de ações, na primeira versão, e prêmio, na segunda versão. Assim, os valores $E(x)$ e $\operatorname{cov}(x, x)$ são valores fixos. Além disso, para a construção da fronteira, valores de $E(M)$ são dados arbitrariamente, e calcula-se o valor $\sigma(M)$, que é o limite inferior do desvio padrão dos candidatos a fator de desconto estocástico, conforme mostrado nas Figuras 1 e 2. O procedimento, então, é:

1) Adotar uma versão de análise;

2) Calcular $E(x)$ e $\operatorname{cov}(x, x)$, de acordo com a versão utilizada;

3) Para um dado valor $E(M)$, calcula-se $b$ segundo a equação (14), para a versão de retornos do índice de ações; ou (17), para a versão de prêmio;

4) A fronteira é construída de acordo com a desigualdade fornecida pela equação (15): $\sigma(M) \geq a b s(b) \sigma(x)$.

Adicionalmente, podemos identificar os valores do coeficiente de aversão relativa ao risco $(\alpha)$ que geram pares $[E(M), \sigma(M)]$ válidos segundo a fronteira. Para tanto, fi- 
xamos o fator de desconto intertemporal da utilidade $(\beta)$ e geramos um conjunto de pares $[E(M), \sigma(M)]$ pela aplicação de valores de aversão ao risco $(\alpha)$, arbitrariamente escolhidos, ao conjunto de dados de consumo. Cada valor de $\alpha$ gera um par $[E(M), \sigma(M)]$, de acordo com a definição de $M$, dada pela equação $M_{t+1} \equiv \beta\left(C_{t+1} / C_{t}\right)^{-\alpha}$. Somente serão considerados válidos os valores de $\alpha$ que gerarem pares acima da reta da fronteira média-variância. Se as estimações econométricas dos parâmetros $\alpha$ e $\beta$ corresponderem a pares $[E(M), \sigma(M)]$ abaixo da fronteira, então o candidato a fator de desconto estocástico não será válido.

\section{APÊNDICE IV - TESTE BASEADO NA FRONTEIRA MÉDIA-VARIÂNCIA DO FATOR DE DESCONTO ESTOCÁSTICO}

Neste apêndice mostramos detalhes sobre a construção e estimação do teste III, conforme Burnside (1994), para a validação dos candidatos a fatores de desconto estocástico por meio das distâncias à fronteira de média-variância de Hansen-Jagannathan.

A idéia central é testar a hipótese nula de que o par $[E(M), \sigma(M)]$ situa-se sobre a fronteira considerada. A alternativa é aceitar que o par não está sobre a fronteira. É necessário saber, então, se o par, na situação de não estar sobre a fronteira, está localizado em seu interior (região em que o candidato a fator de desconto é válido) ou exterior (região em que o candidato a fator de desconto é rejeitado como válido).

Quando apresentamos o ferramental da fronteira de média-variância do fator de desconto estocástico, vimos que o candidato a fator de desconto é expresso por meio de uma regressão, segundo a equação (11). Na primeira versão da fronteira, $x$ representava o retorno dos ativos de risco, e em outra o excesso de retorno sobre ativos livres de risco.

Tomando inicialmente a primeira versão, para o caso de vários ativos de risco, representados pelo vetor $R$, podemos reescrever (11) como:

$$
m=\widehat{R}^{\prime} \theta_{0}+u
$$

em que $\tilde{R}^{\prime}=\left(1 R^{\prime}\right)$. Multiplicado $(20)$ à esquerda por $\tilde{R}$, aplicando o operador esperança, utilizando a condição de ortogonalidade $E(\tilde{R} u)=0$ e a equação (5) sob a forma de média incondicional, temos: 


$$
\theta_{0}=\left[\begin{array}{ll}
E(\widehat{R} & \widehat{R}^{\prime}
\end{array}\right]^{-1}\left(\begin{array}{c}
E m \\
l
\end{array}\right)
$$

em que $l$ é o vetor de 1, que surge do uso da equação (5).

A variância de $m$ é dada por:

$$
\begin{aligned}
& \operatorname{Var}(m)=\theta_{0}^{\prime} \Omega \theta_{0} \\
& \operatorname{com} \Omega=E\left[(\widehat{R}-E \widehat{R})(\widehat{R}-E \widehat{R})^{\prime}\right]
\end{aligned}
$$

A equação (22) é uma versão da equação (15) para o limite inferior da variância do candidato a fator de desconto estocástico. ${ }^{41}$ Cochrane e Hansen (1992) definem o segundo momento de $m$ em torno de zero (momento não centrado). Assim, definindo $M_{R}=\left[E\left(\tilde{R} \widetilde{R}^{\prime}\right)\right]^{-1}$ e usando a equação (21), a equação (22) pode ser reescrita como:

$$
\begin{aligned}
E\left(m^{2}\right) & \geq \theta_{0}^{\prime} E\left(\begin{array}{ll}
\widehat{R} & \widehat{R}^{\prime}
\end{array}\right) \theta_{0} \\
& =\left(\begin{array}{ll}
E m & l^{\prime}
\end{array}\right) M_{R}^{-1}\left(\begin{array}{c}
E m \\
l
\end{array}\right)
\end{aligned}
$$

Definamos os estimadores

$$
\begin{aligned}
& \widehat{M}_{R}=\frac{1}{T} \sum_{t=1}^{T} \hat{R}_{t} \quad \hat{R}_{t}^{\prime} \\
& \hat{M}_{m}=\frac{1}{T} \sum_{t=1}^{T} m_{t}^{2} \\
& \bar{m}=\frac{1}{T} \sum_{t=1}^{T} m_{t}
\end{aligned}
$$

O teste é construído por meio da escolha de $v$ que minimiza a forma quadrática

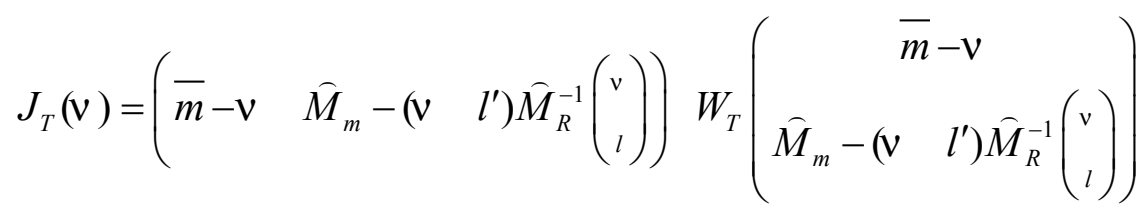

41 Veja, por exemplo, Campbell et al. (1997, Capítulo 8, p. 296-297).

Estud. econ., São Paulo, 36(3): 435-463, jul-set 2006 
em que a matriz $W_{T}$ é escolhida de forma a fornecer o estimador $\widehat{v}$ com o menor desvio padrão assintótico possível. A distância à fronteira eficiente é dada por:

$$
\widehat{\varsigma}=\widehat{M}_{m}-\left(\begin{array}{ll}
v & l^{\prime}
\end{array}\right) \hat{M}_{R}^{-1}\left(\begin{array}{l}
v \\
l
\end{array}\right)
$$

O teste só é realizado se o par $\left[\left(E m, E\left(\mathrm{~m}^{2}\right)\right]\right.$ estiver abaixo da fronteira eficiente, que é o caso $\widehat{\varsigma}<0$. O teste é dado por:

$$
\begin{aligned}
C_{1} & =0, \text { se } \hat{\varsigma} \geq 0 \\
& =T J_{T}, \quad \text { se } \hat{\varsigma}<0
\end{aligned}
$$

Podemos realizar o teste via GMM. As condiçóes de momento são, para o caso de vários ativos:

$$
\begin{aligned}
& E\left[m_{t}-\varphi_{1}\right]=0 \\
& E\left[\begin{array}{ll}
\hat{R}_{t} & \widehat{R}_{t}^{\prime}-\varphi_{2}
\end{array}\right]=0 \\
& E\left[m_{t}^{2}-\left(\begin{array}{ll}
\varphi_{1} & l^{\prime}
\end{array}\right) \varphi_{2}^{-1}\left(\begin{array}{c}
\varphi_{1} \\
l
\end{array}\right)\right]=0
\end{aligned}
$$

Os parâmetros $\varphi_{1}=v$ e $\varphi_{2}=M_{R}$ são obtidos minimizando $J_{T}=g_{T}(\varphi) W_{T} g_{T}(\varphi)$, em que $\varphi=\left(\varphi_{1}, \varphi_{2}\right)$ é:

$$
g_{T}(\varphi)=\left(\begin{array}{c}
\bar{m}-\varphi_{1} \\
\bar{M}_{R}-\varphi_{2} \\
\hat{M}_{m}-\left(\begin{array}{cc}
\varphi_{1} & l^{\prime}
\end{array}\right) \varphi_{2}^{-1}\left(\begin{array}{c}
\varphi_{1} \\
l
\end{array}\right)
\end{array}\right)
$$

No caso da série de prêmio, a equação (21) é modificada, recebendo 0 no lugar de l. Modificando as equaçóes (27), (28) e (30) a (32), chegamos na equação análoga à (33) para a versão envolvendo série de prêmio:

$$
g_{T}(\varphi)=\left(\begin{array}{c}
\bar{m}-\varphi_{1} \\
\widehat{M}_{R}-\varphi_{2} \\
\widehat{M}_{m}-\varphi_{1}^{2} \varphi_{2}^{-1}
\end{array}\right)
$$


Deve-se ressaltar que em nosso trabalho o teste é aplicado para o caso de $R$ formado por um ativo somente: o índice de mercado em questão.

Quando a matriz ótima $W_{T}$ é utilizada, $C_{1}=T J_{T} \sim \chi^{2}$ (\#momentos - \# parâmetros), se $\widehat{\varsigma}<0$. Se $\widehat{\varsigma} \geq 0, C_{1}=0$. O candidato a fator de desconto estocástico será rejeitado para valores grandes de $C_{1}$ e $\widehat{\varsigma}<0$. O GMM descrito acima será realizado se $\widehat{\varsigma}<0$. Neste caso, sob $H_{0}: \varsigma=0$, a estatística $C_{1}$ é distribuída segundo $\chi^{2}$ (1) com probabilidade $1 / 2$, ou seja, a probabilidade correspondente a qualquer valor de $C_{1}$ é dada pela metade da probabilidade usual de uma distribuição $\chi^{2}(1)$. Na prática, devemos dividir por 2 a probabilidade obtida no teste $\chi^{2}$.

Fatores de desconto estocásticos válidos sob o critério da fronteira de média-variância estão associados a $\widehat{\varsigma} \geq 0, \operatorname{com} C_{1}=0$. Assim, uma estatística $T J_{T}$ com p-valor abaixo de 5\% (nível de confiança acima de 95\%) representa pares que não estão sobre a fronteira. Sabendo-se que neste caso o par está abaixo (pois, conforme mencionado, o teste é realizado quando $\bar{\varsigma}<0$ ), o fator é rejeitado.

\section{REFERENCIAS}

Abel, A. Asset prices under habit formation and catching up with the Joneses. American Economic Review, 80, p. 38-42, 1990.

Andrews, D. Heteroskedasticity and autocorrelation consistent covariance matrix estimation. Econometrica, 59, p. 817-858, 1991.

Araújo, E. Entropy-based method of moments test of consumption-based asset pricing models: an application to Brazil. Proceedings do II Encontro da Sociedade Brasileira de Finanças, 2002.

Bansal, R.; Coleman, J. A monetary explanation of the equity premium, term premium, and riskfree rate puzzles'. Journal of Political Economy, 104, p. 1135-1171, 1996.

Bansal, R.; Yaron, A. Risks for the long run: a potential resolution of asset pricing puzzles'. NBER Working Paper N. 8059, 2000.

Barberis, N.; Huang, M.; Santos, T. Propect theory and asset prices'. Quarterly Journal of Economics, 116, p. 1-53, 2001.

Bernartzi, S.; Thaler, R. Myopic loss aversion and the equity premium puzzle'. Journal of Economics, 110, p. 73-92, 1995.

Bonomo, M.; Domingues, G. Os puzzles invertidos no mercado brasileiro de ativos. Finanças aplicadas ao Brasil. Rio de Janeiro, RJ: FGV Editora, 2002. (Marco Bonomo, organizador). 
Burnside, C. Hansen-Jagannathan bounds as classical tests of asset-pricing models'. Journal of Business and Economic Statistics, 12, p. 57-79, 1994.

Campbell, J.; Lo, A.; Mackinley, A. The econometrics of financial markets. Princeton, NJ: Princeton University Press, 1997.

Campbell, J.; Cochrane, J. By force of habit: a consumption-based explanation of aggregate stock market behavior. Journal of Political Economy, 107, p. 205-251, 1999.

Cochrane, J. Asset pricing. Princeton, NJ: Princeton University Press, 2001.

Cochrane, J.; Hansen, L Asset pricing explorations for macroeconomics. NBER Working Paper N. 4088, 1992.

Constantinides, G. Habit formation: a resolution of the equity premium puzzle'. Journal of Political Economy, 98, p. 519-543, 1990.

Constantinides, G.; Duffie, D. Asset prices with heterogeneous consumers. Journal of Political Economy, 104, p. 219-240, 1996.

Constantinides, G.; Donaldson, J.; Mehra, R. Junior can’t borrow: a new perspective on the equity premium puzzle. Quarterly Journal of Economics, 117, p. 269-296, 2002.

Domingues, G. Reproduzindo os momentos dos retornos no Brasil com CAPM intertemporal e utilidade recursiva. 2000. Dissertação (Mestrado), Departamento de Economia. PUC-RJ.

Epstein, L.; Zin, S. Substitution, risk aversion and the temporal behavior of consumption and asset returns: a theoretical framework. Econometrica, 57, p. 937-968, 1989.

. Substitution, risk aversion and the temporal behavior of consumption and asset returns: an empirical investigation. Journal of Political Economy, 99, p. 263$286,1991$.

Garcia, M.; Olivares, G. O prêmio de risco da taxa de câmbio no Brasil durante o Plano Real. Anais do XXI Encontro Brasileiro de Econometria, 1999.

Hansen, L.; Singleton, K. Generalized instrumental variables estimation on nonlinear rational expectations models. Econometrica, v. 50, n. 5, p. 1269-1288, 1982a.

. Errata do artigo generalized instrumental variables estimation on nonlinear rational expectations models. Econometrica, v. 52, n. 1, p. 267-268, 1982 b.

. Stochastic consumption, risk aversion and the temporal behavior of asset returns. Journal of Political Economy, 91, p. 249-268, 1983.

Hansen, L.; Jagannathan, R. Implications of security market data for models of dynamic economies. Journal of Political Economy, 99, p. 225-262, 1991.

Heaton, J.; Lucas, D. Evaluating the effects of incomplete markets on risk sharing and asset pricing. Journal of Political Economy, 104, p. 443-487, 1996. 
. Market frictions, savings behavior and portfolio choice. Journal of Macroeconomic Dynamics, 1, p. 76-101, 1997.

Huang, C.; Litzenberg, R. Foundations of financial economics. New York-Amsterdam: North-Holland Publishing Co., 1988.

Issler, J.; Piqueira, N. Estimating relative risk aversion, the discount rate, and the intertemporal elasticity of substitution in consumption for Brazil using three types of utility function. Brazilian Review of Econometrics, 20, p. 200-238, 2000.

Kreps, D.; Porteus, E. Temporal resolution of uncertainty and dynamic choice theory. Econometrica, 66, p. 185-200, 1978.

Lucas, R. Asset prices in an exchange economy. Econometrica, 46, p. 1429-1445, 1978.

Mankiw, N. The equity premium and the concentration of aggregate shocks. Journal of Financial Economics, 17, p. 211-219, 1986.

McGrattan, E.; Prescott, E. Taxes, regulations, and asset prices. Working Paper N. 610, Federal Reserve Bank of Minneapolis, 2001.

Mehra, R. The equity premium: why is it a puzzle? NBER Working Paper N. w9512, 2003.

Mehra, R.; Prescott, E. The equity premium: a puzzle. Journal of Monetary Economics, 15, p. 145-161, 1985.

Nakane, Márcio; Soriano, L. Real balances in the utility function: evidence for Brazil. Working Paper N. 68, Banco Central do Brasil, 2003.

Newey, W.; West, K. A simple, positive-definite, heteroskedasticity and autocorrelation consistent covariance matrix. Econometrica, 55, p. 703-708, 1987.

. Automatic lag selection in covariance matrix estimation. Review of Economic Studies, 61, p. 631-653, 1994.

Obstfeld, M.; Rogoff, K. Foundations of international macroeconomics. Cambridge, Massachussets: MIT Press, 1996.

Sampaio, F. Existe o equity premium puzzle no Brasil? Finanças aplicadas ao Brasil. In: Bonomo, M. (org.), Os puzzles invertidos no mercado brasileiro de ativos. Finanças aplicadas ao Brasil. Rio de Janeiro, RJ: FGV Editora, 2002.

Soriano, A. Testando CCAPM com dados brasileiros. 1999. Dissertação (Mestrado), Departamento de Economia, PUC-RJ.

. Testando o CCAPM através das fronteiras de volatilidade e da equação de Euler. Finanças aplicadas ao Brasil. In: Bonomo, M. (org.), Os puzzles invertidos no mercado brasileiro de ativos. Finanças aplicadas ao Brasil. Rio de Janeiro, RJ: FGV Editora, 2002. 
Storesletten, K.; Telmer, C.; Yaron, A. Asset pricing with idiosyncratic risk and overlapping generations. Working Paper. Pittsburgh: Carnegie Mellon University, 1999.

Weil, P. The equity premium puzzle and riskfree rate puzzle. Journal of Monetary Economics, 24, p. 401-421, 1989.

Yoshino, J. The second-best asset pricing for solving both the equity premium puzzle and the low interest rate puzzle. V Encontro Brasileiro de Finanças. São Paulo, 2005 . 